\title{
Alimentation en eau et assainissement Rapport général
}

\section{Water supply and sanitation - General report}

\author{
Daniel Villessot \\ Directeur à la Direction Technique et de la Recherche, Suez Lyonnaise des Eaux
}

After a brief recall on the principal international recent contracts obtained by the French huge companies, this general report is relative to the different management methods developed. The second part of this general report will be published later on.

\section{INTRODUCTION}

Depuis près de vingt ans maintenant, les Groupes français privés, qui ont acquis en France un savoir-faire plus que centenaire pour la gestion déléguée des services publics de l'alimentation en eau potable et de l'assainissement, ont entamé le développement de leurs activités à l'international. Du fait des succès remportés dans un contexte de concurrence internationale grandissante, l'image de ces Groupes est devenue progressivement internationale puis mondiale aujourd'hui. L'Ecole Française de l'Eau était à l'origine de ces succès ; elle a permis leur multiplication et du fait de l'acquisition de références nouvelles, l'expérience de ces Groupes est aujourd'hui mondiale.

Diverses contributions présenteront les détails et particularismes de ce que nous avons voulu être un inventaire certes non exhaustif, mais représentatif des diverses situations rencontrées de gestion déléguée, ainsi que des solutions techniques innovantes apportées.

La mondialisation quasi-simultanée des réseaux des centres de recherche des deux plus grands Groupes illustre parfaitement cette internationalisation de l'Ecole française de l'Eau pour sans cesse se rapprocher des exploitations en vue d'apporter au plus vite expertise et soutien aux structures d'exploitation d'une part, mais aussi tirer enseignements et bénéfices des implantations locales et des structures de recherche déjà existantes et reconnues.
Les rapports généraux préparés par les deux Rapporteurs à partir des expériences décrites dans chaque cas spécifique ont pour objectif de démontrer et valoriser ces caractéristiques aujourd'hui reconnues à travers le monde. Ce savoir-faire et les résultats obtenus attirent de plus en plus souvent des compétiteurs étrangers de diverses origines plus ou moins proches du secteur de l'Eau : Ingénieurs Conseils, services publics autres (Energie par exemple), ou même entreprises de génie civil, ou encore fournisseurs d'équipements et de matériels pour le secteur. L'expérience acquise depuis plus d'un siècle en France, et depuis plusieurs années à l'international permet à nos Groupes d'entretenir un leadership certain mais de plus en plus contesté. Ainsi que les figures 1 et 2 le montrent, les deux Groupes sont aujourd'hui présents sur les cinq continents et servent en eau et/ou assainissement une population totale proche de 150 millions de personnes.

\section{LA GRANDE ADAPTABILITÉ DE L'OFFRE DE GESTION DE SERVICES PUBLICS}

Depuis toujours les Groupes ont su offrir aux Collectivités Locales françaises maîtres d'ouvrages, dans le cadre de contrats de diverses natures, des solutions parfaitement adaptées au contextes locaux, politique, technique ou économique. Ainsi est née une grande diversité de contrats depuis la Régie intéressée, l'affermage jusqu'à la Concession, avec des nuances multiples et variées pour toujours adapter l'offre 
aux situations locales spécifiques. La conséquence est le développement dans le cadre des sociétés d'une forte aptitude à la négociation de contrats " sur mesure ", adaptés à la demande du Client, négociés dans un cadre de très forte concurrence où donc la recherche du mieux disant est permanente ; même limités dans le temps, ces contrats comportent une obligation de durée dans la qualité des prestations ; leur renouvellement y est souvent lié ; toutefois une trop courte durée interdirait toute possibilité d'investissement avec des temps de retour à moyen et long termes, habituels dans le cas de services publics.

Particularité de l'Ecole Française de l'Eau, la pratique de ce mode contractuel souple et adaptable a été rapidement internationalisée et il est intéressant de ce point de vue de constater qu'elle a permis de créer des références remarquables pour nos Groupes. Citons quelques exemples dont certains sont détaillés par les contributions de cette session : - des contrats ont été négociés de gré-à-gré entre les Groupes français et le Maître d'ouvrage étranger ; c'est ainsi que le premier contrat de Lyonnaise des Eaux en Asie du Sud-Est a été signé à MACAO en 1985, et que plus récemment celui de JAKARTA a été obtenu avec les Autorités locales avant d'être validé par la Banque Mondiale. Le champ couvert peut être celui de l'eau et de l'assainissement. - des contrats de gestion simplifiée, souvent désignés dans les pays anglo-saxons comme contrats $\boldsymbol{O \& M}$ (Operation and Maintenance) ; c'est le cas des contrats signés avant 1997 aux Etats -Unis à INDIANAPOLIS ; depuis 1997, une amélioration des dispositions réglementaires américaines permet des contrats de durée plus longue, pouvant aller jusqu'à 15 ans et favorables au financement, lorsque nécessaire, de l'amélioration ou de l'extension des infrastructures. - des contrats d'affermage, véritable particularisme de l'Ecole Française de l'Eau, dont il est toujours difficile de faire comprendre les particularismes (notamment en matière d'investissement) à nos interlocuteurs étrangers, et ce... même dans leur langue !

- sur la base des contrats de concession ont été développés des contrats de type BOT (Build Operate and Transfer) ou encore BOOT (Build Operate Own and Transfer) pour répondre à des demandes particulières. Dans l'une ou l'autre de ces catégories, qu'il n'est pas question de détailler ici, figurent les contrats signés en Allemagne, Argentine, Australie, Malaisie, Mexique dont il sera donné plus de détails dans les contributions des différents auteurs.

Enfin les Groupes français ont pu participer à la privatisation des structures nationales par achat des nouvelles structures mises en place ; l'exemple le plus significatif reste encore aujourd'hui celui de l'Angleterre où les trois Groupes ont réalisé dès 1985 des investissements importants ayant permis pour certains, des remodelages par voie d'acquisitions nouvelles. Mais cette politique a aussi permis de découvrir dans un secteur totalement privatisé, les particularismes de gestion avec des Régulateurs dont les missions, fixées par le Gouvernement, sont complémentaires les unes aux autres ; ainsi, OFWAT (Office for Water Services), DWI (Drinking Water Inspectorate) et EA (Environment Agency) ont des actions dont les particularismes sont décrits dans la contribution de H. SPEED.

\section{III — L'EFFICACITÉ DES SOLUTIONS PROPOSÉES}

En général, les solutions apportées lors d'une prise nou- velle d'exploitation, dans l'un ou l'autre des cadres contractuels décrits ci-dessus, mettent en cuvre des originalités de structures et de moyens de gestion, et des innovations technologiques relatives soit aux procédés de traitement ou d'épuration des eaux, soit à leur conduite souvent très automatisée, soit encore à des moyens innovants de réalisation et de gestion des réseaux d'alimentation en eau potable ou de collecte des eaux usées.

Ces apports multiples sont réalisés et maîtrisés par des Hommes de l'Art qui ont souvent contribué à leur développement. Cela constitue une exception française au regard des expertises étrangères et notamment des ingénieries anglosaxonnes, souvent limitées à un rôle de prescripteur n'exerçant plus aucun contrôle technique ou financier sur la gestion quotidienne des infrastructures ainsi développées.

S'inscrire dans la longévité pour apporter et garantir des solutions efficaces aux problèmes posés, voilà bien une qualité première des techniques offertes et issues de l'Ecole Française de l'Eau. Certaines des contributions à ces Journées de l'Hydraulique illustrent plus particulièrement ces apports ; dans ce cadre, nous pouvons mentionner la contribution à l'alimentation en eau de Sydney (Australie) apportée par le Groupe Suez Lyonnaise des Eaux qui, à l'issue d'un appel d'offre international, a conçu installé et mis en service dans des délais records ce qui est aujourd'hui la plus grande unité de production d'eau potable au monde, réalisée en une seule étape, pour produire 3 millions de $\mathrm{m}^{3}$ en première étape et 4,2 millions de $\mathrm{m}^{3}$ si l'on prend en compte son extension future. La contribution de P. MAZOUNIE et al. souligne les principales innovations techniques et l'architecture informatique mise à disposition des opérateurs.

Autre exemple, toujours en Australie, où depuis janvier 1996, United Water International (UWI) filiale du Groupe VIVENDI a pris en charge la gestion et l'exploitation d'usines de traitement d'eau potable, d'eaux usées, de réseaux de distribution d'eau et de collecte d'eaux usées dans le cadre d'un contrat de 15 années avec pour objectif d'améliorer le service au consommateur, de diminuer les coûts d'exploitation et de mettre en place une structure locale de recherche et développement ; la contribution de P. SZTAJNBOK décrit les progrès de UWI dans ces divers domaines.

La gestion du service des eaux de la ville d'AGUASCALIENTES (Mexique) présentée par J. COUTELLE, ou encore celle des 26 usines de traitement d'eau potable d'une capacité totale de production proche de 1 million de $\mathrm{m}^{3}$ par jour de l'état de Sélangor (Malaysie) décrite par J.M.SEILLIER, la gestion de l'eau et de l'assainissement de ROSTOCK, première concession du secteur privé en Allemagne fédérale, détaillée par P. BUTZ sont autant d'exemples réussis de ces transferts multiples de savoir-faire en matière de gestion privée de services publics et de développement simultané, pour les besoins du contrat, de technologies performantes.

Les réseaux de distribution d'eau potable et les réseaux de collecte des eaux usées sont également l'objet de travaux importants ; leurs hydrauliques, leurs fonctionnements, l'optimisation et l'actualisation des coûts, la modélisation des concentrations en produits non réactifs ou réactifs sont autant de sujets de préoccupation pour le gestionnaire en étroite liaison avec les chercheurs ainsi qu'il est décrit par Y. KADAR et al.(MEKOROT), M.MARTAUD (SAFEGE), P. CHOPARD et al.(SEDIF), E. FROSSARD et al. (AGUAS ARGENTINAS) et par ailleurs dans la partie R\&D de ce 
rapport général. Une mention particulière pour le cas des réseaux d'assainissement de BUENOS AIRES (Argentine) ; S. DOAN VAN et al. décrivent la solution originale apportée pour le curage des grands collecteurs dits “ Cloaca Maxima " (sections comprises entre 2,30 et 4,00 mètres, longueurs de plusieurs kilomètres, en charge 24 heures sur 24 , profondément enfouis, encombrés à $70 \%, \ldots)$, dans le centre ville de la capitale ; la mise au point de matériels et de techniques adaptés a été réalisée sur un centre d'essais en France reconstituant à l'échelle 1 la problématique rencontrée localement.

\section{IV — L'APPUI TECHNIQUE ET LA FORMA- TION DES ÉQUIPES LOCALES}

Il est encore remarquable de constater que l'ensemble de ces activités est mené à bien avec un nombre limité de personnels expatriés ; certes, ceux qui participent localement à ces expériences remarquables sont des Professionnels ayant acquis, sur des exploitations françaises, un savoir-faire et des compétences techniques et de gestion de contrats qui en font des Experts aguerris et chevronnés capables de surmonter bien des difficultés ! Encore leur faut-il, arrivés sur sites, s'adapter aux cultures locales, parler une langue qui peut souvent leur être étrangère, et savoir se faire intégrer au personnel en place qui peut redouter toutes sortes de mesures compte tenu du changement du mode de gestion ou des nouvelles technologies qui seront mises en place.

Il faut donc une expérience forte de ces modes de gestion pour pouvoir en assurer le transfert et les nécessaires adaptations aux contextes locaux. A tous les niveaux, du niveau politique au niveau des personnels d'exploitation sans oublier les Clients domestiques et industriels, il faut expliquer, convaincre et démontrer que les solutions originales qui sont ou seront mises en place sont celles permettant d'atteindre des objectifs économiques, techniques et de qualité de services que d'autres auparavant, n'ont peut être pas pu (su ?) réaliser...

Outre les qualités personnelles de ces Professionnels, ils doivent pouvoir compter sur un appui technique jamais défaillant des structures centrales, parfois lointaines. Les Experts, spécialistes de telle ou telle technique, méthode, ou responsable de gestion comptable ou financière, sont souvent appelés pour réaliser des missions de soutien technique de plus ou moins courte durée dès le démarrage des nouveaux contrats ; ils participent totalement au transfert et à l'adaptation des savoir-faire et des techniques. Au contact de leurs collègues et des personnels en postes, ils réaliseront aussi des missions de formation sur sites indispensables à un meilleur partage des valeurs qui constituaient le modèle français : une organisation efficace de l'ensemble des personnels dans des structures clairement établies, la maîtrise des techniques de production, le souci de la qualité du produit et des services fournis aux Clients. Plusieurs des présentations soulignent l'importance de la prise en compte de ce paramètre : la formation des personnels locaux, l'intégration des cadres locaux avec ceux expatriés en vue de constituer une internationalisation des équipes de direction. L'exemple rapporté par J.M. SEILLIER et al. ( Malaisie) détaille particulièrement ce souci.

H. SPEED souligne combien aussi le modèle français peut encore dans le domaine du contrôle de gestion s'enrichir au contact d'autres exemples tels que celui de l'Angleterre où le rôle joué par les Régulateurs est de nature différente de ce que connaît la France, mais tend à être adapté à d'autres pays ou contrats.

\section{D LA PRISE EN COMPTE DU CLIENT FINAL}

Maillon longtemps sous-estimé dans la gestion française des services de l'eau et de l'assainissement, le Client final, domestique ou industriel est devenu aujourd'hui l'objet de toutes les attentions. Afin de lui fournir la qualité du service outre la qualité de l'eau en harmonie avec ses aspirations, $\mathrm{H}$. SPEED et J. MAWBRAY décrivent dans leurs contributions, comment la privatisation des compagnies d'eau en Angleterre a créé des attentes et des besoins nouveaux de la part des Clients ; lorsque pour satisfaire à ces besoins en Angleterre se sont additionnés des besoins exprimés par Lyonnaise des Eaux dans le cadre d'autres contrats, des projets nouveaux sont nés tel celui de développer un logiciel commun de gestion des clientèles - ICIS. Depuis lors, même dans le cadre de contrats récents, l'expérience montre que les exigences des Clients croissent très rapidement et qu'il est indispensable de pouvoir disposer de systèmes évolutifs conçus pour couvrir les besoins du prochain millénaire.

L'amélioration du service fourni au Client final est désormais fréquemment listée parmi les termes de références à respecter dans les nouveaux contrats ; des difficultés subsistent toutefois pour s'accorder sur une définition univoque et aisément paramétrable du service fourni ; c'est l'objet de recherches en cours.

Une autre attention de la part des sociétés vis-à-vis de son Client s'exprime par la mise en auvre de nouvelles approches techniques qui permettent de réduire les nuisances liées aux travaux en sites urbains sur les réseaux (travaux sans tranchées ; limitation des nuisances - odeur, bruit, emprise au sol - lors des curages de réseaux d'assainissement) notamment dans les sites dont le trafic automobile est souvent fort congestionné.

Enfin, la prise en compte du Client final revêt un caractère très innovant lorsqu'il s'agit d'alimenter en eau potable et d'assurer l'assainissement de populations des quartiers défavorisés des mégalopoles des pays émergeants ; ainsi que le décrit Th. CHAMBOLLE les habituelles techniques mises en œuvre de façon conventionnelle en France comme ailleurs en Europe doivent être totalement reconsidérées dans ces cas pour pouvoir intégrer d'autres aspects à caractère plus sociologique mais absolument indispensables si l'on veut trouver des solutions acceptées, partagées et donc respectées dès leur mise en cuvre par ces populations.

\section{VI $\square$ LA RECHERCHE PERMANENTE DE SOLUTIONS INNOVANTES ET PERFORMANTES}

L'ensemble des contributions à ces 25 èmes Journées de l'Hydraulique ne peut constituer qu'un aperçu limité des enjeux majeurs mais aussi des succès déjà acquis par les Groupes français dans le cadre du développement de leurs activités mondiales. Nous le savons, de nouvelles affaires ont été acquises depuis que nous avons écrit ces contributions et heureusement, mais pas fortuitement, les difficultés restent peu nombreuses, passagères, et souvent liées à des contextes politiques ayant évolué après la signature du contrat.

Il n'est pas surprenant dans un tel environnement que cette réussite des Groupes français fasse naître des velléités fortes 
de sociétés étrangères du même secteur d'activité ou de segments voisins tels que celui de l'ingéniérie ou encore de la distribution d'énergie, pour se positionner sur ce marché.

Il est donc nécessaire de rechercher en permanence de nouvelles solutions encore plus innovantes et toujours plus performantes pour continuer ce développement international. C'est l'objet de la Recherche et du Développement, et il n'est pas surprenant de constater que les plus importants budgets de R\&D consacrés au domaine de l'eau et de l'assainissement par des sociétés privées le sont par des Groupes français. Quelques éléments de leurs approches et de leurs programmes sont développés par M.M. BOURBIGOT. Ils soulignent un pas important réalisé ces derniers mois par l'un et l'autre des deux grands Groupes : l'internationalisation de leurs structures de R\&D et l'implantation de structures délocalisées à proximité des nouvelles exploitations pour d'une part être proche des réalités du terrain mais aussi d'autre part pour tirer avantages des technologies et savoir-faire recensés ici et là, à travers le monde.

L'Ecole Française de l'Eau qui se trouvait à l'origine de ce formidable développement de nos sociétés à travers le monde, a permis leur internationalisation. L'expérience ainsi acquise peut être croisée avec de nouveaux savoir-faire tirés de ces implantations quelquefois lointaines ; le résultat doit être australien en Australie, américain aux Etats-Unis, argentin en Argentine, etc. comme il a su être anglais en Angleterre ; il doit contribuer à la mondialisation de nos Groupes dont l'encadrement, les structures et les moyens liés doivent encore évoluer pour mieux s'inscrire dans ce nouveau cadre. 